\title{
Prediction of HIV-1 protease cleavage site using a combination of sequence, structural, and physicochemical features
}

\author{
Onkar Singh and Emily Chia-Yu Su* \\ From The 27th International Conference on Genome Informatics \\ Shanghai, China. 3-5 October 2016
}

\begin{abstract}
Background: The human immunodeficiency virus type 1 (HIV-1) aspartic protease is an important enzyme owing to its imperative part in viral development and a causative agent of deadliest disease known as acquired immune deficiency syndrome (AIDS). Development of HIV-1 protease inhibitors can help understand the specificity of substrates which can restrain the replication of HIV-1, thus antagonize AIDS. However, experimental methods in identification of HIV-1 protease cleavage sites are generally time-consuming and labor-intensive. Therefore, using computational methods to predict cleavage sites has become highly desirable.
\end{abstract}

Results: In this study, we propose a prediction method in which sequence, structural, and physicochemical features are incorporated in various machine learning algorithms. Then, a bidirectional stepwise selection algorithm is incorporated in feature selection to identify discriminative features. Further, only the selected features are calculated by various encoding schemes and used as input for decision trees, logistic regression, and artificial neural networks. Moreover, a more rigorous three-way data split procedure is applied to evaluate the objective performance of cleavage site prediction. Four benchmark datasets collected from previous studies are used to evaluate the predictive performance.

Conclusions: Experiment results showed that combinations of sequence, structure, and physicochemical features performed better than single feature type for identification of HIV-1 protease cleavage sites. In addition, incorporation of stepwise feature selection is effective to identify interpretable biological features to depict specificity of the substrates. Moreover, artificial neural networks perform significantly better than the other two classifiers. Finally, the proposed method achieved 80.0\% 97.4\% in accuracy and $0.815 \sim 0.995$ evaluated by independent test sets in a three-way data split procedure.

Keywords: HIV-1 protease, Cleavage sites, Sequence features, Structural features, Physicochemical properties, Pseudo amino acid composition, Machine learning

\section{Background Introduction}

In early 1980's, human immunodeficiency virus (HIV) and acquired immune deficiency syndrome (AIDS) transition began in perishing modus with a leading cause of death. AIDS is an advanced stage infection with the HIV [1]. The first AIDS cases were reported in the United

\footnotetext{
* Correspondence: emilysu@tmu.edu.tw

Graduate Institute of Biomedical Informatics, College of Medical Science and

Technology, Taipei Medical University, Taipei, Taiwan
}

States in June 1981 by Center for Disease Control (CDC) [2]. Now it has been 35 years and still HIV is one of the major global public health issues. According to global HIV statistics, 36.9 million people had HIV and 1.2 million people died from AIDS-related illnesses (UNAIDS, 2015). However, after the confrontation with AIDS epidemic, unprecedented endeavors have been coordinated towards the advancement of antiretroviral treatments of AIDS that assault and repress the action of HIV-1 protease (HIV-1 PR). 
HIV-1 protease is the principle etiologic agent of AIDS discovered by Gallo and coworkers in 1984 [3]. It is able to infect and destroy the human immune system, and allows life threating infection. HIV-1 PR, a homodimeric enzyme belonging to aspartate family also known as aspartyl retropepsin, plays a crucial role in viral maturation [4]. HIV constructs many of its protein in one long piece consisting of several tandemly linked proteins. HIV-1 PR has a responsibility to cleave Gag and Gag-Pol polyproteins into their component proteins responsible for the maturation of new virions, which can then infect new cells [5]. Thus, an HIV-specific protease is necessary for the HIV to make more functional viruses. Without HIV-1 PR, it is not possible for HIV to replicate due to unavailability of infectious virion and it remains uninfected. HIV-1 protease specifically binds with a precursor protein in octapeptide length, and cleaves it at the scissile bond represented as P4-P3-P2-P1 $\downarrow$ P1' -P2'-P3'$\mathrm{P} 4$ ', where $\mathrm{N}$-terminus as $\mathrm{Pm}$ (i.e., nonprime-side) and C-terminus as Pm' (i.e., prime-side) in perceived octapeptide arrangement, though “ $\downarrow$ ” signifies a nearness of a scissile bond [5].

\section{Challenges of HIV-1 protease identification}

HIV-1 PR is the key target of the most effective antiviral drugs for the treatment of HIV-1 infection as it processes the viral precursor Gag-Pol polyproteins, and allows maturation of the immature virion to make more functional viruses. However, the specificity of HIV-1 PR is partially understood because viral polyproteins do not share sequence homology and binding motifs between various substrates [5]. At present, researchers have partially succeeded to develop HIV protease inhibitors that are accessible for HIV treatment. However, they have conditional drawbacks such as poor bioavailability and excruciating infectiousness [6] that lead researchers to proceed with their endeavors to create novel and more potent compounds. Also, due to the tremendous amount of potential peptides, it is difficult to discover inhibitors by ordinary ways to deal with testing various types of peptides one by one, which is more labor-intensive and time-consuming.

\section{Previous work in HIV-1 protease cleavage site prediction}

To conquer the difficulties to identify HIV protease, researchers are inclined towards in-silico approaches to predict HIV-1 protease cleavage sites [7]. In the past few years, several previous studies incorporated biological features with machine learning algorithms and gained better predictive performance compared to traditional approaches. You et al. [8] incorporated machine learning algorithms including artificial neural network (ANN) and support vector machine (SVM) to examine the specificity of an HIV-1 protease for the discovery and development of effective protease inhibitors. Kontijevkis et al. used an extensive dataset collected from HIV proteome research, and designed a rule-based predictive model on rough sets to analyze the specificity of HIV-1 protease [5]. Kim et al. organized their own datasets by compiling peptide sequences, and used a combination of neural networks and decompositional approaches to generate symbolic rules [9]. Ogul et al. used variable context markov chains (VCMC) to develop a generative model for HIV-1 cleavage specificity, and suggested that VCMC model is effective for prediction of cleavage sites of all proteases [10]. Nanni et al. developed a robust and reliable system in which genetic programming was used to design encoding techniques, and they showed the proposed ensemble method performed better than nonoptimized SVM with standard encoding by cross-validation [11]. Jaeger et al. proposed a new fusion technique in which they added several classifiers including decision trees (DT), ANN, and SVM. They used cross-validation for evaluation and reported that the combined method achieved better performance than using a single classifier [12]. Kim et al. introduced a new feature selection method with multilayer perceptron (MLP) and used a decompositional approach to trained MLP. Li et al. developed a theoretical framework based on kernel methods to reduced dimensionality with linear support vector machine (LSVM) classifiers [13]. Newell proposed a new cascade detection algorithm to study the specificity on two datasets, and reported that the proposed method is useful in detection of multifactor synergies in several datasets [14]. Gök and Özcerit used OETMAP encoding schemes based on amino acid features together with linear classifiers. The encoding schemes improved prediction performance compared to standard amino acid encodings evaluated on two datasets by crossvalidation [15]. Song and coworkers developed a protease specificity prediction server to predict unique substrates and their cleavage sites. They used support vector regression and bi-profile Bayesian feature extraction method to predict cleavage sites [16]. Niu et al. studied protease specificity correlation-based feature subset (CfsSubset) selection method combined with genetic algorithms [17]. Bozek et al. developed a model for structure-based prediction of HIV tropism and identification of important V3 loop properties for coreceptor usage [18]. Rögnvaldsson et al. proposed a method to combine linear support vector machine with orthogonal encoding schemes. They claimed that the model is effective for predicting cleavage sites by HIV-1 protease [19]. Liu et al. used feed forward back propagation neural network in their method along with feature selection schemes [20]. 


\section{Specific aims of this study}

The advancement of reasonable HIV protease inhibitors can happen when we have a robust and suitable technique for anticipating the cleavage sites in proteins by HIV protease [7]. In this study, we propose a prediction method in which sequence, structural, and physicochemical features are incorporated in various machine learning algorithms. For feature selection, a bidirectional stepwise selection algorithm is incorporated to identify the discriminative features. Then the features are encoded by various encoding schemes and used as input for decision trees, logistic regression, and artificial neural networks. Moreover, a more rigorous three-way data split is applied to evaluate the objective performance of cleavage site prediction. The proposed HIV-1 protease specificity prediction method can further help the development of more potential HIV-1 protease inhibitors.

\section{Methods \\ Datasets}

In the present study, four benchmark datasets organized by Rögnvaldsson et al. [19] were used in our proposed method. The benchmark datasets are collections of octamers containing cleavage and non-cleavage sites as shown in Table 1. The 746, 1625, Schilling, and Impens datasets contain 746 (401 cleaved and 345 non-cleaved), 1625 (374 cleaved and 1251 non-cleaved), Schilling (434 cleaved and 2838 non-cleaved), and Impens (149 cleaved and 798 non-cleaved) octamers, respectively. The datasets are available in the supplementary material [Additional files 1, 2, 3 and 4].

\section{Feature extraction}

Amino acids are the essential components of peptides and proteins, and each of 20 amino acids has unique but different properties. The combination of the properties of various residues within a protein can influence diversification and characteristics of the protein structure and function. The aim of the study is to develop a better prediction model using various combinations of features that can predict the HIV-1 protease cleavage sites. The present investigation involved extraction of three different kinds of features based on sequence,

Table 1 Four benchmark datasets for HIV-1 cleavage site prediction

\begin{tabular}{llll}
\hline Datasets & Octamers & Cleavage sites & Non-cleavage sites \\
\hline 746 & 746 & 401 & 345 \\
1625 & 1625 & 374 & 1251 \\
Schilling & 3272 & 434 & 2838 \\
Impens & 947 & 149 & 798 \\
\hline
\end{tabular}

structure, and physicochemical properties. Several feature extraction methods in propy 1.0 software package [11] were employed to extract sequence-based and physicochemical-based features. For structure-based feature extraction, NetSurfP [21] web server was used. Besides, we considered AAindex [22] database for physicochemical properties. This database contains numerical indices which represent several physicochemical and biochemical properties of amino acids and amino acid pairs.

\section{Sequence-based features}

Sequence-based features include the composition of amino acids which contains 20 factors with each representing the occurrence frequency of one native amino acid in a given peptide. The selected sequence based features are amino acid composition (AAC), dipeptide composition (DipC), pseudo amino acid composition (PseAAC) [23]. AAC and its variations have been demonstrated that they are influential in predicting HIV-1 protease cleavage sites. Besides, protease has a preference for some amino acid compositions at their cleavage sites. For example, trypsin recognizes essential amino acid lysine and arginine and cleaves at carboxyl terminal. Afterward, the DipC was selected to represent occurrence frequencies of amino acid pairs in peptides. At last, the important point about PseAAC is that it is endowed with the information about AAC and also contains information beyond it, and hence can better reflect the features of peptides through a discrete model. In our study, 20 and 400 variables are used to represent AAC and DipC, respectively. Another 25 variables were utilized for PseAAC by propy 1.0 package.

\section{Structure-based features}

Structure-based features are important to study the substrate specificity of the HIV-1 protease with the aim of obtaining a better differentiation between cleavage and non-cleavage sites. Also, it was stated in statistics for structural and sequence comparisons of protein pairs that the structural comparison can explore almost double as many different relationships as sequence comparison [24]. In this work, two structure-based features including solvent accessibility (SA) and secondary structure elements (SSE) were selected. Through solvent accessibility, we can depict the exposed surface of the entire protein or individual amino acid. The significance of proper surface presentation of cleavage sites in the solvent-exposed region for efficient proteolysis is well evidenced. We thus predicted solvent accessibility using NetSurfP web server, and three columns were selected to represent the accessibility of a peptide, including buried or exposed (B/E) class, relative solvent accessibility (RSA), and absolute surface accessibility (ASA) for each 
residue in a peptide. Another structure-based feature we used in this study was the secondary structure which is characterized by folding of a peptide chain into the $\alpha$ helixes, $\beta$-sheets, or random coils. The caspase substrate analysis indicated the considerable proportion of the cleavage sites located in $\alpha$-helixes and $\beta$-sheets [16]. NetSurfP web server [21] was used for the secondary structure prediction, and generated three columns of probabilities for $\alpha$-helix, $\beta$-sheet, and random coil for each residue in a peptide.

\section{Physicochemical property features}

Each peptide and proteins are the combinations of twenty amino acid components. These amino acids have common constituents of the amine groups, carboxyl groups, and side chains which have several functional groups, and these functional groups are responsible for distinct physical properties of each amino acid. In the study, we selected six physicochemical properties including hydrophobicity, polarizability, steric property, isoelectric point, volume, and polarity. Hydrophobicity is a physical property of amino acids representing the tendency of water to exclude non-polar molecules. Moreover, as stated earlier in previous studies, the hydrophobic nature of cleavage sites can efficiently bind with the substrates by Van der Waals interaction and help identify cleavage and non-cleavage sites in peptides [17]. The ability to form instant dipoles known as polarizability, through which the dynamical response of the closed system to external fields can be determined, and provide perception about a molecule's internal structure can also be made [25]. Steric properties can be appraised by the attributes of an atom within the molecule. The overlapping electron clouds lead to the repulsion when the atoms are brought close to each other. A steric property encompasses various effects such as steric hindrance, steric shielding, steric attractions, chain crossing, and steric inhibition of resonance. These properties are largely responsible for the shapes (i.e., conformation) of molecules and also reactivity [26]. The isoelectric point can be defined as the $\mathrm{pH}$ at which amino acid is neutral [27]. Volume, a standard feature of native protein structures, is the dense packing of amino acid residues within interior regions, and a key parameter in understanding packing is the volume that individual amino acid residue occupies in different environments [28]. Polarity confers molecules and compounds with distinctive features regarding the structure and chemical interaction with other molecules. Due to this property, polar amino acids are exposed on the surface of proteins [29].

\section{Machine learning algorithms}

In this study, three algorithms have been applied to predict the HIV-1 protease specificity, including DT, ANN, and logistic regression (LR). The sequence, structural, and physicochemical features are incorporated as inputs for DT, ANN, and LR to investigate discriminative biological features and construct an accurate predictive model. The descriptive and predictive modeling provides insights that drive better decision-making. Keeping this in mind, our research group was motivated to use SAS Enterprise Miner Workstation 13.2, having the suite of machine learning algorithms that enables to create accurate predictive and descriptive model. It also allowed us to compare several predictive models simultaneously. ANN, a machine learning approach resembling the biological neural network especially human brain, is fabricated to mimic the structure and function of our nervous system. It scores over the conventional rule-based programming owing to its broad applicability for the various tasks such as classification, sequence recognition, and novelty detection [24]. The important aspect about ANN is a non-parametric model while most statistical methods are parametric models that need the higher background of statistics. Moreover, ANN generates models to detect non-linear interactions between dependent and independent variables. DT is a simple yet effective machine learning algorithm to yield interpretable results with numerous conceivable results. It orders examples by shorting them down the tree from the root hub to leaf hub to arranging the cases [30]. There is a distinct advantage of applying decision trees to classify biomedical problems that make DT better predictors among others. The best characteristic of using trees is very intuitive and easy to explain. In addition, variable nonlinearity usually results in poor predictive performance while using other classifiers such as simple regressions. Another advantage of DT is that nonlinearity property in data does not influence the predictive performance of DT. Therefore, DT predictors can be applicable to data with nonlinear relationships. On the other hand, LR is a machine learning algorithm where the dependent variable is categorical. It calculates the probability of categorical dependent variable and other independent variables. The most important point of $L R$ is that the expected values of response variables are modeled based on the combination of values taken by the predictors [31]. Our motivation to use LR is a white-box model that allows an interpretation of model parameters. It gives real probabilities of predicted class unlike DT and SVM and it is easier to update the model to take in new data with the help of online gradient descendent method.

\section{System architecture}

The system architecture of our proposed method for predicting cleavage sites in HIV-1 protease is illustrated 
in Fig. 1. The analysis workflow involves several steps, including query protein peptide input, feature extraction, feature selection, machine learning algorithms, and the prediction results. First, octamers are extracted from query protein sequences by a sliding window of size eight. Then, the sequence features, structural features, and physicochemical properties were extracted and encoded with the aid of propy 1.0 software package. Further, a bidirectional stepwise selection algorithm is incorporated to select only the discriminative biological features as input to be submitted to machine learning algorithms for prediction. At last, examination of all models was made, and the execution of prediction model was illustrated. The proposed method is named as ProCleSSP (Protease Cleavage site prediction based on Sequence, Structural, and Physicochemical features).

\section{Evaluation measures}

Model assessment is critical in regards to measuring the nature of predictions. In our experiments, we incorporated a three-way data split procedure to avoid overfitting and overestimation, and randomly divide our raw data into $90 \%$ as the training set, $5 \%$ as the validation set, and $5 \%$ as the test set. The training set is used to train a predictive model. The validation dataset is applied for feature selection and parameter tuning. The test dataset is incorporated as an independent set only to evaluate the real performance of a prediction method. As for the evaluation measures, we used accuracy and area under the receiver operating characteristics (ROC) curve to compare with other previous studies. The accuracy (Acc.) of a prediction method is calculated as the summation of true positives and true negatives divided by the total number of data. In addition, the area under the ROC curve (AUC) is used to assess performance during parameter selection, and is one of the most appropriate measures of performance as it is nonparametric and threshold independent. It is calculated from ROC curve which is a fundamental tool for diagnostic test evaluation. In an ROC curve, the true positive rate (i.e., sensitivity) is plotted in function of the false positive rate (i.e., 1-specificity) for different cutoff points of a parameter. In our study, we use AUC as an evaluation measure to select a combination of effective features and classifiers. Other metrics are also computed to allow more comprehensive evaluation of predictive performance. Sensitivity (Sen.) and specificity (Spe.) measure how well a classifier detects cleavage

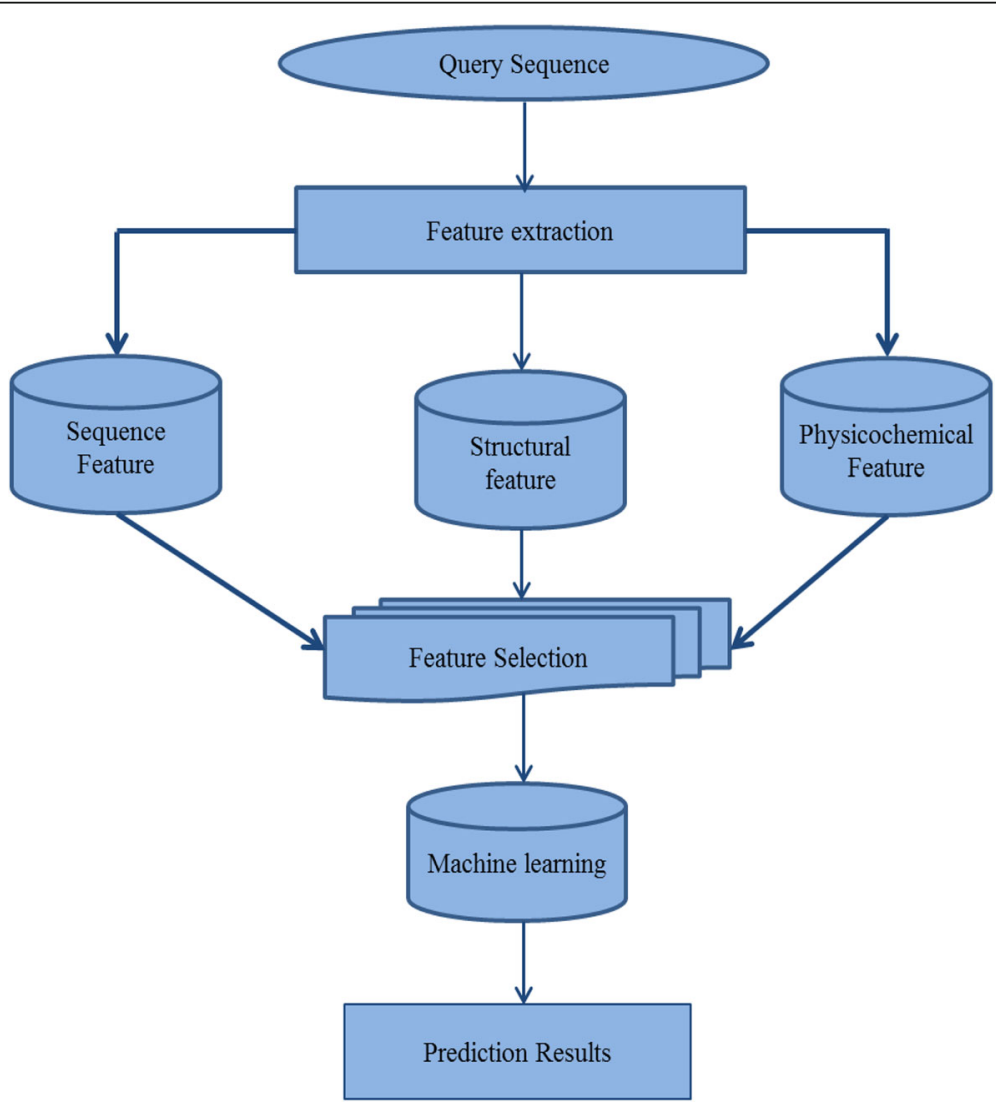

Fig. 1 System architecture of the proposed method 
sites as cleavage sites and non-cleavage sites as noncleavage sites, respectively. The following equations define these statistics, where TP, TN, FP, and $F N$ denote the numbers of true positives, true negatives, false positives, and false negatives, respectively.

$$
\begin{gathered}
\text { Sen. }=\frac{T P}{T P+F N} \\
\text { Spe. }=\frac{T N}{T N+F P} \\
\text { Acc. }=\frac{T P+T N}{T P+T N+F P+F N}
\end{gathered}
$$

\section{Results and discussion}

In ProCleSSP, the biological features are extracted from sequence-based, structure-based, and physicochemical properties. Then the extracted biological features from the four benchmark datasets (i.e., 746, 1625, Schilling, and Impens) are used as input features to three machine learning algorithms (i.e., ANN, DT, and LR), and predictive performance are optimized by AUC based on the validation set instead of the test set to avoid overfitting. Here, to compare the effects of various biological features, the predictive performance is analyzed by single feature type prediction and hybrid feature type prediction. For single feature type prediction, the performance of sequence-based features, structure-based features, and physicochemical properties are compared. In addition, the hybrid feature type prediction are conducted by the combination of various feature types, including sequence and structure features, sequence and physicochemical features, structure and physicochemical features, and all three types of features.

\section{Prediction performance based on single feature types}

In our experiment, the effects of different biological features are compared individually. The prediction performance based on sequence features, structural features, and physicochemical features are detailed in the following sections.

\section{Sequence-based features}

Three types of sequence-based features (i.e., AAC, DipC, and PseAAC) are used to depict the effect of using sequence patterns to distinguish cleavage sites from noncleavage sites. The predictive performance based on sequence features for the four benchmark datasets is shown in Table 2. We compare the accuracy and AUC of different algorithms based on AAC, DipC, PseAAC, and the combination of all three compositions. Experiment results show that incorporation of DipC performed better than using AAC or PseAAC itself. This suggests that DipC could be a better indicator to predict HIV-1 protease cleavage sites due to its capability to consider

\begin{tabular}{|c|c|c|c|c|c|c|}
\hline \multirow[t]{2}{*}{ Features } & \multicolumn{2}{|l|}{$\underline{\text { DT }}$} & \multicolumn{2}{|l|}{$\underline{L R}$} & \multicolumn{2}{|l|}{ ANN } \\
\hline & Acc.(\%) & $A \cup C$ & Acc.(\%) & AUC & Acc.(\%) & AUC \\
\hline \multicolumn{7}{|c|}{746 Dataset } \\
\hline AAC & 83.7 & 0.897 & 86.4 & 0.938 & 81.0 & 0.935 \\
\hline DipC & 75.6 & 0.793 & 86.4 & 0.865 & $\underline{91.9}$ & 0.974 \\
\hline PseAAC & 78.3 & 0.787 & 86.4 & 0.938 & 81.0 & 0.885 \\
\hline Seq_All & 78.3 & 0.831 & 86.4 & 0.847 & $\underline{91.9}$ & $\underline{0.979}$ \\
\hline \multicolumn{7}{|c|}{1625 Dataset } \\
\hline AAC & 91.4 & 0.908 & 84.1 & 0.904 & 91.4 & 0.952 \\
\hline DipC & 92.6 & 0.861 & 96.3 & 0.972 & $\underline{98.7}$ & $\underline{0.987}$ \\
\hline PseAAC & 90.2 & 0.822 & 87.8 & 0.921 & 87.8 & 0.945 \\
\hline Seq_All & 92.6 & 0.882 & 96.3 & 0.958 & $\underline{98.7}$ & 0.984 \\
\hline \multicolumn{7}{|c|}{ Schilling Dataset } \\
\hline AAC & 87.7 & 0.664 & 86.5 & 0.856 & 88.9 & 0.858 \\
\hline DipC & 87.7 & 0.526 & 87.1 & 0.806 & $\underline{89.5}$ & 0.790 \\
\hline PseAAC & 87.1 & 0.500 & 86.5 & $\underline{0.864}$ & 88.3 & 0.858 \\
\hline Seq_All & 87.7 & 0.611 & 87.7 & 0.802 & 87.1 & 0.821 \\
\hline \multicolumn{7}{|c|}{ Impens Dataset } \\
\hline AAC & 85.1 & 0.500 & 80.8 & 0.857 & 89.3 & 0.886 \\
\hline DipC & 85.1 & 0.500 & 82.9 & 0.579 & $\underline{93.6}$ & $\underline{0.893}$ \\
\hline PseAAC & 87.2 & 0.721 & 78.7 & 0.814 & 87.2 & 0.868 \\
\hline Seq_All & 87.2 & 0.802 & 85.1 & 0.696 & 89.3 & 0.875 \\
\hline
\end{tabular}

Table 2 Predictive performance of sequence features for the four benchmark dataset

pairwise amino acid pair relationships. For the machine learning algorithms, ANN achieved better predictive performance except for the AUC of the Schilling dataset.

\section{Structure-based features}

Two structure-based features, SA and SSE, were incorporated individually or combined together to identify cleavage sites in our study. For solvent accessibility, we used three descriptors, including solvent accessibility class (i.e., exposed or buried), RSA, and ASA. For secondary structure, the probability of $\alpha$-helix, $\beta$-sheet, and random coil are predicted by the NetSurfP web server. An octapeptide generates 24 descriptors for each of solvent accessibility and secondary structure features. The predictive performance using structural features for the four benchmark data sets is shown in Table 3. The results indicate that SA usually performed better than SSE when it is used individually or combined with SSE. This lends support on our assumption that the cleavage sites usually occur on the surface of a molecule and thus SA serves as an effective factor to identify cleavage sites in HIV-1 protease. When we compare machine learning algorithms, ANN also performed better than the others. 
Table 3 Predictive performance of structural features for the four benchmark datasets

\begin{tabular}{|c|c|c|c|c|c|c|}
\hline \multirow[t]{2}{*}{ Features } & \multicolumn{2}{|l|}{ DT } & \multicolumn{2}{|l|}{$\underline{L R}$} & \multicolumn{2}{|l|}{ ANN } \\
\hline & Acc.(\%) & AUC & Acc.(\%) & $A \cup C$ & Acc.(\%) & AUC \\
\hline \multicolumn{7}{|c|}{746 Dataset } \\
\hline SSE & 62.1 & 0.626 & 59.4 & 0.715 & 78.3 & 0.838 \\
\hline SA & 83.7 & 0.791 & 78.4 & 0.771 & 81.0 & 0.771 \\
\hline Str_All & $\underline{83.7}$ & 0.791 & 70.2 & 0.806 & 78.4 & $\underline{0.897}$ \\
\hline \multicolumn{7}{|c|}{1625 Dataset } \\
\hline SSE & 81.7 & 0.756 & 76.8 & 0.673 & 85.3 & 0.742 \\
\hline SA & 91.4 & 0.920 & 89.0 & 0.961 & $\underline{96.3}$ & $\underline{0.977}$ \\
\hline Str_All & 91.5 & 0.920 & 85.4 & 0.936 & 89.0 & 0.935 \\
\hline \multicolumn{7}{|c|}{ Schilling Dataset } \\
\hline SSE & 87.1 & 0.500 & 88.3 & 0.775 & 88.3 & 0.800 \\
\hline SA & $\underline{89.5}$ & 0.788 & 84.0 & 0.828 & 87.1 & 0.840 \\
\hline Str_All & 89.5 & 0.788 & 83.4 & 0.824 & 85.8 & $\underline{0.843}$ \\
\hline \multicolumn{7}{|c|}{ Impens Dataset } \\
\hline SSE & 85.1 & 0.500 & 85.1 & 0.729 & 87.2 & 0.761 \\
\hline SA & 89.3 & 0.736 & 89.3 & 0.918 & $\underline{95.7}$ & $\underline{0.950}$ \\
\hline Str_All & 87.2 & 0.571 & 89.3 & 0.857 & 89.3 & 0.914 \\
\hline
\end{tabular}

"The best accuracy and AUC in each dataset are underlined

\section{Physicochemical features}

In physicochemical properties, six properties including hydrophobicity, polarizability, steric properties, isoelectric point, volume, and polarity are incorporated to detect cleavage sites. Each property was encoded as 25 descriptors by PseAAC using propy 1.0 software package. The physicochemical properties were examined individually as well as in combinations. The predictive performance based on physicochemical properties for the benchmark datasets is shown in Table 4.

\section{Prediction performance based on hybrid feature types}

In our study, the prediction performance based on hybrid features is undertaken. Hybrid features denote the combinations of sequence, structure, and physicochemical features. The combinations of such features could contain more extensive information than the single feature types. In this section, four distinct combinations are used to explore the impact of the properties in protease cleavage site prediction. First, sequence and structure features are combined together to check whether these properties influenced the cleavage sites. The number of features obtained for this combination is 493 (i.e., 445 for sequence and 48 for structure features). Secondly, sequence and physicochemical features were consolidated, a total of 595 features were combined (i.e., 445 for sequence features and 150 physicochemical properties). Thirdly, structure and physicochemical features are combined and generated 198 features (i.e., 48 for
Table 4 Predictive performance of physicochemical property features for the four benchmark datasets

\begin{tabular}{|c|c|c|c|c|c|c|}
\hline \multirow[t]{2}{*}{ Features } & \multicolumn{2}{|l|}{ DT } & \multicolumn{2}{|l|}{$L R$} & \multicolumn{2}{|l|}{ ANN } \\
\hline & Acc.(\%) & AUC & Acc.(\%) & AUC & Acc.(\%) & AUC \\
\hline \multicolumn{7}{|l|}{746 Dataset } \\
\hline Hydrophobicity & 75.6 & 0.735 & 83.7 & 0.956 & 89.1 & $\underline{0.968^{*}}$ \\
\hline Steric property & 89.1 & 0.929 & 86.4 & 0.941 & 81.0 & 0.932 \\
\hline Polarizability & 81.0 & 0.815 & 83.7 & 0.953 & 83.7 & 0.947 \\
\hline Isoelectric point & 81.0 & 0.865 & 86.4 & 0.953 & 83.7 & 0.953 \\
\hline Polarity & 83.7 & 0.838 & 83.7 & 0.912 & 86.4 & 0.909 \\
\hline Volume & 83.7 & 0.838 & 54.0 & 0.500 & 54.0 & 0.500 \\
\hline Phy_All & 84.9 & 0.882 & 93.6 & 0.885 & 97.3 & 0.953 \\
\hline \multicolumn{7}{|l|}{1625 Dataset } \\
\hline Hydrophobicity & 87.8 & 0.849 & 84.1 & 0.896 & 86.5 & 0.874 \\
\hline Steric property & 91.4 & 0.897 & 85.3 & 0.896 & 91.4 & 0.934 \\
\hline Polarizability & 93.9 & 0.914 & 87.8 & 0.936 & $\underline{96.3}$ & 0.957 \\
\hline Isoelectric point & 91.4 & 0.918 & 82.9 & 0.914 & 93.9 & 0.968 \\
\hline Polarity & 86.5 & 0.847 & 87.8 & 0.904 & 89.0 & 0.919 \\
\hline Volume & 92.6 & 0.896 & 89.0 & 0.933 & 93.9 & $\underline{0.974}$ \\
\hline Phy_All & 92.7 & 0.882 & 92.7 & 0.921 & 92.7 & 0.944 \\
\hline \multicolumn{7}{|l|}{ Schilling Dataset } \\
\hline Hydrophobicity & 87.7 & 0.708 & 89.5 & 0.862 & 89.5 & 0.863 \\
\hline Steric property & 88.3 & 0.721 & 86.5 & 0.837 & 88.3 & 0.843 \\
\hline Polarizability & 89.5 & 0.683 & 89.5 & 0.854 & $\underline{90.8}$ & 0.853 \\
\hline Isoelectric point & 88.3 & 0.733 & 87.7 & 0.858 & 89.5 & 0.860 \\
\hline Polarity & 87.1 & 0.500 & 87.1 & 0.860 & 88.3 & 0.865 \\
\hline Volume & 88.9 & 0.622 & 88.3 & 0.847 & 88.3 & 0.810 \\
\hline Phy_All & 88.9 & 0.593 & 89.5 & $\underline{0.876}$ & 85.2 & 0.863 \\
\hline \multicolumn{7}{|l|}{ Impens Dataset } \\
\hline Hydrophobicity & 85.1 & 0.500 & 80.8 & 0.686 & 87.2 & 0.886 \\
\hline Steric property & 89.3 & 0.845 & 82.9 & 0.825 & 89.3 & 0.893 \\
\hline Polarizability & 85.1 & 0.500 & 85.1 & 0.864 & 89.3 & 0.943 \\
\hline Isoelectric point & 85.1 & 0.500 & 78.7 & 0.850 & $\underline{93.6}$ & $\underline{0.982}$ \\
\hline Polarity & 85.1 & 0.500 & 85.1 & 0.743 & 82.9 & 0.682 \\
\hline Volume & 85.1 & 0.500 & 85.1 & 0.736 & 80.8 & 0.500 \\
\hline Phy_All & 91.5 & 0.839 & 82.9 & 0.796 & 87.2 & 0.839 \\
\hline
\end{tabular}

"The best accuracy and AUC in each dataset are underlined

structure and 150 for physicochemical features). The last combination is to combine all three feature types together, and yields a total of 643 features. The predictive performance of the four combinations for the benchmark dataset is illustrated in Table 5. For the feature combinations, it is frequently observed that combining multiple features together can compensate the properties of various biological features and further improve the predictive performance in terms of both accuracy and AUC. The only exception is the Impens dataset in which 
Table 5 Predictive performance of hybrid features for the four benchmark datasets

\begin{tabular}{|c|c|c|c|c|c|c|}
\hline \multirow[t]{2}{*}{ Features } & \multicolumn{2}{|l|}{ DT } & \multicolumn{2}{|l|}{$\underline{L R}$} & \multicolumn{2}{|l|}{ ANN } \\
\hline & Acc.(\%) & AUC & Acc.(\%) & $A \cup C$ & Acc.(\%) & $A \cup C$ \\
\hline \multicolumn{7}{|l|}{746 Dataset } \\
\hline Seq + Str & 78.3 & 0.788 & 91.8 & 0.982 & 94.5 & $\underline{0.994^{*}}$ \\
\hline Seq + Phy & 83.7 & 0.838 & 86.4 & 0.968 & 94.5 & 0.976 \\
\hline Phy + Str & 83.7 & 0.810 & 78.3 & 0.860 & 91.8 & 0.982 \\
\hline Seq + Str + Phy & 75.6 & 0.841 & $\underline{97.2}$ & 0.991 & $\underline{97.2}$ & 0.988 \\
\hline \multicolumn{7}{|l|}{1625 Dataset } \\
\hline Seq + Str & 89.0 & 0.910 & 96.3 & 0.980 & 95.1 & $\underline{0.992}$ \\
\hline Seq + Phy & 89.0 & 0.785 & 97.5 & 0.958 & $\underline{98.7}$ & 0.990 \\
\hline Phy + Str & 91.4 & 0.940 & 86.5 & 0.810 & 93.9 & 0.985 \\
\hline Seq + Str + Phy & 91.4 & 0.956 & 95.1 & 0.980 & 97.5 & 0.990 \\
\hline \multicolumn{7}{|l|}{ Schilling Dataset } \\
\hline Seq + Str & 90.8 & 0.845 & 86.5 & 0.865 & $\underline{92.0}$ & 0.873 \\
\hline Seq + Phy & 87.1 & 0.500 & 90.8 & 0.837 & 88.9 & 0.825 \\
\hline Phy + Str & 85.1 & 0.500 & 80.8 & 0.603 & 80.8 & 0.596 \\
\hline Seq + Str + Phy & 88.9 & 0.810 & 89.5 & 0.826 & 91.4 & $\underline{0.895}$ \\
\hline \multicolumn{7}{|l|}{ Impens Dataset } \\
\hline Seq + Str & 89.3 & 0.682 & 89.3 & 0.918 & $\underline{93.6}$ & 0.918 \\
\hline Seq + Phy & 91.4 & 0.839 & 87.2 & 0.889 & 91.4 & 0.896 \\
\hline Phy + Str & 85.1 & 0.500 & 82.9 & 0.889 & $\underline{93.6}$ & $\underline{0.932}$ \\
\hline Seq + Str + Phy & 87.2 & 0.675 & 87.2 & 0.889 & 89.3 & 0.850 \\
\hline
\end{tabular}

incorporation of solvent accessibility performed slightly better than any combination from Table 5 . In addition, it is also interesting to observe that ANN and LR perform significantly better than DT when different types of features are combined as input for prediction of cleavage sites. This suggests that incorporation of more advanced machine learning algorithms, such as ANN, could be a better choice to identify discriminative features from heterogeneous data.

\section{Best combinations of features and algorithms for each dataset}

In our experiment, we used the AUC of the validation dataset to select a best combination of features and algorithms for each dataset, and then incorporated the test set to show the objective performance of cleavage site prediction in HIV-1 protease. The best combinations of features and algorithms for each dataset are listed in Table 6 and the ROC plots are shown in Figure S1-S4 of the supplementary material [Additional file 5]. Experiment results show that ProCleSSP achieved AUC of $0.994,0.992,0.895$, and 0.950 based on validation sets for the 746, 1625, Schilling, and Impens datasets, respectively. We also attain accurate prediction accuracy of
94.5\%, 95.1\%, 91.4\%, and 95.7\% for the 746, 1625, Schilling, and Impens datasets, respectively. The sensitivity and specificity range from $57.1 \% \sim 100 \%$ and $88.2 \% \sim 100 \%$ for the validation sets, respectively. This suggests that imbalanced datasets for cleavage site identification could result in the observation that our method achieves higher specificity compared to sensitivity. However, if an independent test set, which has not been used to construct the classifier or tune features and parameters, is incorporated to evaluate the most objective performance of the prediction method, our results demonstrated that the performance could often be overestimated. For the feature selection, our method suggested that the best feature set is the combination of sequence and structural features together for the 746 and 1625 datasets. For the largest Schilling dataset, incorporation of all sequence, structural, and physicochemical features performed the best. For the Impens dataset, our validation results select SA as the best set of feature. However, it is interesting to notice that there is a large difference between validation and test performance. This might be resulted from the fact that the number of SA features is much smaller than others and thus this instability could lead to inadequate for prediction. On the other hand, ANN performed consistently better than the other two machine learning algorithms for prediction of HIV-1 protease cleavage sites. We incorporated a rigorous three-way data split procedure to prevent overfitting in our experiments, while most previous studies incorporated internal validation or cross-validation for performance evaluation. For example, ProCleSSP achieved slightly better performance (i.e., 95.1\% in accuracy and 0.992 in AUC) in the 1625 dataset compared to performance (i.e., 93.0\% in accuracy and 0.940 in AUC) in Kontijevskis et al. In addition, when compared with state-of-the-art method by Rögnvaldsson et al., their approach performed better than our method. Although ProCleSSP only attains comparable or slightly better performance compared with other approaches, a more objective performance of cleavage site prediction is illustrated in the proposed method.

\section{Interpretable biological features for cleavage site identification}

In the proposed method, we incorporated several machine learning algorithms to predict cleavage sites. Although it has been demonstrated that ANN achieved the best predictive performance, discriminative biological features for cleavage sites can be interpretable by algorithms such as DT or LR. The interpretable models give a closed form of approximation of variables where the importance of each variable is explicit. Here, we draw attention to the decision tree model and variable importance for each dataset. In Fig. 2, the decision tree model for the 746 dataset based on Seq + Str features represents a hierarchal segmentation of the data. The original 
Table 6 Predictive performance based on selected features and machine learning algorithms based on validation sets and test sets

\begin{tabular}{lllllll}
\hline Datasets & Features & Algorithm & Sen.(\%) & Spe.(\%) & Acc.(\%) & AUC \\
\hline 746 & Seq + Str & ANN & $100.0(100.0)^{*}$ & $88.2(94.4)$ & $94.5(97.4)$ & $0.994(0.995)$ \\
1625 & Seq + Str & ANN & $94.7(89.4)$ & $95.2(96.8)$ & $95.1(95.1)$ & $0.992(0.994)$ \\
Schilling & Seq + Str + Phy & ANN & $57.1(27.3)$ & $96.5(95.8)$ & $91.4(86.6)$ & $0.895(0.815)$ \\
Impens & SA & ANN & $71.4(44.4)$ & $100.0(89.8)$ & $95.7(80.0)$ & $0.950(0.816)$ \\
\hline
\end{tabular}

${ }^{*}$ The predictive performance of test set is shown in parenthesis

segment is the entire dataset, also known as the root node of the tree, and it is first portioned into two or more segments by applying a series of simple rules. Each rule assigns an observation on a segment based on the value of an explanatory variable for that observation. For example, the decision tree model first selects the RSA_4 variable (i.e., the RSA of the $4^{\text {th }}$ position in the octapeptide) as the first rule to distinguish cleavage sites and non-cleavage sites. If the RSA_4 value of an octamer is greater than or equal to 0.4055 , we follow the right subtree; otherwise, the rules in the left subtree are applied. In a similar fashion, each resulting segment is further

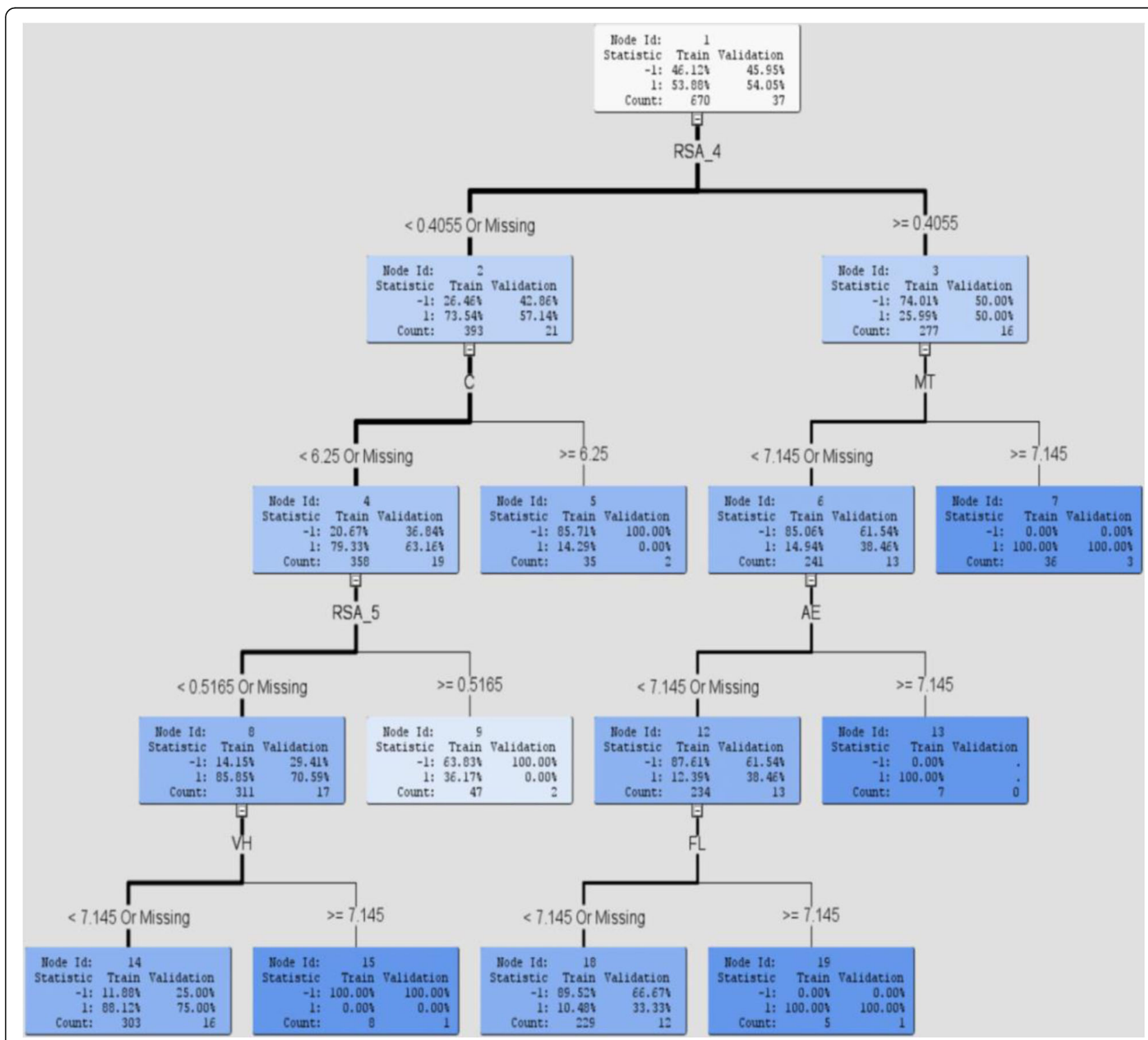

Fig. 2 Decision tree of the 746 dataset based on Seq + Str features 
portioned into sub-segments, and each sub-segment is further portioned into more sub-segments. From the right subtree of the previous example, the second rule selected by decision tree model to identify cleavage sites is MT (i.e., the DipC of methionine and threonine in the octapeptide). When MT is greater than or equal to 7.145 , the octapeptide is predicted as cleavage site in the right subtree; otherwise, rules from the left subtree are applied for further partition. This process continues until no more portioning is possible. This process of segmenting is called recursive portioning, and it results in a hierarchy of segments within segments. The decision trees for the other three datasets are illustrated in Figure $\mathrm{S} 5-\mathrm{S} 7$ of the supplementary material [Additional file 6]. In Table 7, variable importance of the decision tree model in Fig. 2 is ranked by reduction of Gini index for the training set. The top ranked variables RSA_4 and RSA_5 variables corresponded well with the findings that solvent accessibility served as a discriminative feature to predict cleavage sites [16]. Moreover, the selected RSA_4 and RSA_5 variables suggested that the centered position 4 and position 5 in the octapeptide play a crucial role for identification of cleavage sites, and these two positions have also been illustrated important in a traditional classification of HIV-1 protease substrates [5]. This lends support on our assumption that our method can identify important biological features to identify cleavage sites. In addition, combinations of hydrophobic and polar amino acid dipeptides (i.e., $\mathrm{MT}, \mathrm{VH}, \mathrm{AE}$, and $\mathrm{FL}$ ), which can form hydrogen bonds with others, are selected as important features preferred for cleavage sites [32].

\section{Conclusions}

To predict protease cleavage site, the understanding of HIV-1 protease specificity becomes imperative. In this study, we demonstrated that the combination of various sequence, structure, and physicochemical features can play a vital role in the identification of HIV-1 protease cleavage sites and understanding of the specificity of the substrates. We incorporated three machine learning algorithms to compare the predictive performance of

Table 7 Interpretable biological features selected by decision tree model based on Seq + Str features for the 746 dataset

\begin{tabular}{lllc}
\hline Rank & Variable & Description & Importance \\
\hline 1 & RSA_4 & RSA at the $4^{\text {th }}$ position of an octamer & 1.0000 \\
2 & MT & DipC of methionine \& threonine & 0.7855 \\
3 & C & AAC of cysteine & 0.6060 \\
4 & RSA_5 & RSA at the $5^{\text {th }}$ position of an octamer & 0.5238 \\
5 & VH & DipC of valine \& histidine & 0.4059 \\
6 & AE & DipC of alanine \& glutamic acid & 0.3769 \\
7 & FL & DipC of phenylalanine \& leucine & 0.3268 \\
\hline
\end{tabular}

protease cleavage sites. Experiment results suggested that the hybrid biological features performed better than the single feature types. In addition, the results also lend support on our assumption that incorporation of various biological features can compensate each other and achieve more accurate performance. Moreover, through this study, we can identify an effective set of feature combinations that help identify the highly favorable sites where cleavage events take place. The source codes and datasets are freely available for download as standalone software from the link provided here (https://drive.google.com/ open?id=0B-_hwmxkV77wNlY0cUxoQmcyOWc).

\section{Additional files}

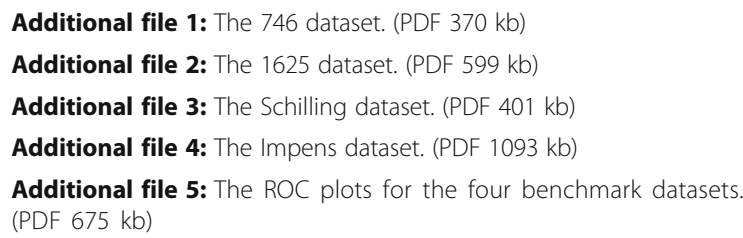

Additional file 6: Decision trees of the 1625, Schilling, and Impens datasets. (PDF 464 kb)

\section{Acknowledgments}

We thank Prof. Jia-Ming Chang, Prof. Yuan-Chii Gladys Lee, Prof. Hung-Wen Chiu, and Prof. Chun-Chang Chen for their helpful suggestions and valuable comments.

\section{Declarations}

This article has been published as part of BMC Bioinformatics Volume 17 Supplement 17, 2016: Proceedings of the 27th International Conference on Genome Informatics: bioinformatics. The full contents of the supplement are available online at http://bmcbioinformatics.biomedcentral.com/articles/ supplements/volume-17-supplement-17.

\section{Funding}

The research was supported in part by Ministry of Science and Technology under grant MOST104-2221-E-038-010-MY2 to Emily Chia-Yu Su.

\section{Availability of data and materials}

All data generated or analyzed during this study are included in the supplementary information of this published article.

\section{Authors' contributions}

OS implemented the system, analyzed the data, and drafted the manuscript. ECYS designed the method, supervised the project, and revised the manuscript. Both authors read and approved the final manuscript.

\section{Competing interests}

The authors declare that they have no competing interests.

\section{Consent for publication}

Not applicable.

Ethics approval and consent to participate

Not applicable.

Published: 23 December 2016

\section{References}

1. Weiss RA. How does HIV cause AIDS? Science. 1993;260(5112):1273-9.

2. Brik A, Wong $\mathrm{CH}$. HIV-1 protease: mechanism and drug discovery. Org Biomol Chem. 2003;1(1):5-14. 
3. Gallo RC, Montagnier L. The discovery of HIV as the cause of AIDS. N Engl J Med. 2003;349(24):2283-5.

4. Verkhivker GM. Coarse-Grained Modeling of the HIV-1 Protease Binding Mechanisms: II. Folding Inhibition. In: Computational Intelligence Methods for Bioinformatics and Biostatistics. Springer Berlin Heidelberg; 2009. p. 13-24.

5. Kontijevskis A, Wikberg JE, Komorowski J. Computational proteomics analysis of HIV-1 protease interactome. Proteins. 2007;68(1):305-12.

6. Lv Z, Chu Y, Wang Y. HIV protease inhibitors: a review of molecular selectivity and toxicity. HIV AIDS (Auckl). 2015;7:95-104.

7. Chou KC. Prediction of human immunodeficiency virus protease cleavage sites in proteins. Anal Biochem. 1996:233(1):1-14.

8. You L, Garwicz D, Rognvaldsson T. Comprehensive bioinformatic analysis of the specificity of human immunodeficiency virus type 1 protease. J Virol. 2005;79(19):12477-86.

9. Kim H, Zhang Y, Heo Y-S, Oh H-B, Chen S-S. Specificity rule discovery in HIV1 protease cleavage site analysis. Comput Biol Chem. 2008;32(1):72-9.

10. Ogul H. Variable context Markov chains for HIV protease cleavage site prediction. Bio Systems. 2009;96(3):246-50.

11. Nanni L, Lumini A. Using ensemble of classifiers for predicting HIV protease cleavage sites in proteins. Amino Acids. 2009;36(3):409-16.

12. Jaeger $\mathrm{S}$, Chen S-S. Information fusion for biological prediction. J Data Sci. 2010;8(2):269-88.

13. Li X, Hu H, Shu L. Predicting human immunodeficiency virus protease cleavage sites in nonlinear projection space. Mol Cell Biochem. 2010;339(1-2):127-33.

14. Newell NE. Cascade detection for the extraction of localized sequence features; specificity results for HIV-1 protease and structure-function results for the Schellman loop. Bioinformatics. 2011;27(24):3415-22.

15. Gök M, Özcerit AT. A new feature encoding scheme for HIV-1 protease cleavage site prediction. Neural Comput \& Applic. 2012;22(7):1757-61.

16. Song J, Tan H, Perry AJ, Akutsu T, Webb GI, Whisstock JC, Pike RN. PROSPER: an integrated feature-based tool for predicting protease substrate cleavage sites. PLoS One. 2012;7(11):e50300.

17. Niu B, Yuan XC, Roeper P, Su Q, Peng CR, Yin JY, Ding J, Li H, Lu WC. HIV-1 protease cleavage site prediction based on two-stage feature selection method. Protein Pept Lett. 2013;20(3):290-8.

18. Bozek K, Lengauer T, Sierra S, Kaiser R, Domingues FS. Analysis of physicochemical and structural properties determining HIV-1 coreceptor usage. PLoS Comput Biol. 2013;9(3):e1002977.

19. Rognvaldsson T, You L, Garwicz D. State of the art prediction of HIV-1 protease cleavage sites. Bioinformatics. 2015;31(8):1204-10.

20. Liu H, Shi X, Guo D, Zhao Z. Feature selection combined with neural network structure optimization for HIV-1 protease cleavage site prediction. BioMed Res Int. 2015;2015: Article ID 263586.

21. Petersen B, Petersen TN, Andersen P, Nielsen M, Lundegaard C. A generic method for assignment of reliability scores applied to solvent accessibility predictions. BMC Struct Biol. 2009;9:51.

22. Kawashima S, Kanehisa M. AAindex: amino acid index database. Nucleic Acids Res. 2000;28(1):374.

23. Chou KC. Some remarks on protein attribute prediction and pseudo amino acid composition. J Theor Biol. 2011;273(1):236-47.

24. Levitt M, Gerstein M. A unified statistical framework for sequence comparison and structure comparison. Proc Natl Acad Sci U S A. 1998;95(11):5913-20

25. Zhou L, Lee F, Wilcox W, Christensen J. Magnetic polarizability of hadrons from lattice QCD. Nucl Phys B Proc Suppl. 2003;1 19:272-4.

26. Weinhold F. Chemistry: A new twist on molecular shape. Nature. 2001; 411(6837):539-41.

27. Nelson $\mathrm{DL}$, Lehninger $\mathrm{AL}$, Cox MM. Lehninger principles of biochemistry. Macmillan: Cambridge University Press, New York; 2008.

28. Counterman AE, Clemmer DE. Volumes of individual amino acid residues in gas-phase peptide ions. J Am Chem Soc. 1999;121(16):4031-9.

29. Wilson $\mathrm{K}$, Walker J. Principles and techniques of biochemistry and molecular biology. Cambridge University Press; 2010.

30. Narayanan A, Wu X, Yang ZR. Mining viral protease data to extract cleavage knowledge. Bioinformatics. 2002;18 suppl 1:S5-13.

31. You L. Detection of cleavage sites for HIV-1 protease in native proteins. Comput Syst Bioinformatics Conf. 2006; 5:249-56.

32. Prabu-Jeyabalan M, Nalivaika E, Schiffer CA. Substrate shape determines specificity of recognition for HIV-1 protease: analysis of crystal structures of six substrate complexes. Structure. 2002;10(3):369-81.

\section{Submit your next manuscript to BioMed Central and we will help you at every step:}

- We accept pre-submission inquiries

- Our selector tool helps you to find the most relevant journal

- We provide round the clock customer support

- Convenient online submission

- Thorough peer review

- Inclusion in PubMed and all major indexing services

- Maximum visibility for your research

Submit your manuscript at www.biomedcentral.com/submit
Biomed Central 